\title{
L’alliance originale de la coopération et de la mutualité : l'école de Saint-Claude
}

\section{A unique alliance of the cooperative and mutual movements: The Saint-Claude School \\ La escuela de Saint-Claude: alianza original de la cooperación y la mutualidad}

\section{Stève Desgré}

Numéro 327, janvier 2013

URI : https://id.erudit.org/iderudit/1015151ar

DOI : https://doi.org/10.7202/1015151ar

Aller au sommaire du numéro

Éditeur(s)

Association Recma

ISSN

1626-1682 (imprimé)

2261-2599 (numérique)

Découvrir la revue

Citer cet article

Desgré, S. (2013). L’alliance originale de la coopération et de la mutualité :

l'école de Saint-Claude. Revue internationale de l'économie sociale, (327), 75-87. https://doi.org/10.7202/1015151ar
Résumé de l'article

Dans l'histoire de l'économie sociale, l'école de Saint-Claude représente l'exemple même d'une coopération à vocation sociale. A la fin du xixe siècle, les coopérateurs militants de ce pôle industriel jurassien s'investissent dans la mutualité. La Fraternelle et la Mutuelle de la maison du peuple oeuvrent main dans la main. Cette expérience locale reste cependant une exception à l'échelle nationale qui mérite une analyse plus approfondie. Comment expliquer que coopération et mutualité, soeurs de l'économie sociale, ne se soient pas alliées plus souvent ? L'analyse des facteurs théoriques, historiques et juridiques qui caractérisent leurs points communs et leurs différences est nécessaire, pour mettre en perspective l'originalité de cette expérience. Les dirigeants socialistes de la coopérative d'alimentation La Fraternelle de Saint-Claude sont devenus acteurs de l'action sanitaire et sociale dans le Haut-Jura et ont fondé des structures mutualistes. Le facteur juridique a joué un rôle essentiel dans cette évolution structurelle. 


\section{L'ALLIANCEORIGINALEDELACOOPÉRATION ET DE LA MUTUALITÉ: L'ÉCOLE DE SAINT-CLAUDE}

*Enseignant, université de Nantes, départementLEA, doctorant. Mél.: steve.desgre@univ-nantes.fr. par Stève Desgré*

Dans l'histoire de l'économie sociale, l'école de Saint-Claude représente l'exemple même d'une coopération à vocation sociale. A la fin du XIX siècle, les coopérateurs militants de ce pôle industriel jurassien s'investissent dans la mutualité. La Fraternelle et la Mutuelle de la maison du peuple ouvrent main dans la main. Cette expérience locale reste cependant une exception à l'échelle nationale qui mérite une analyse plus approfondie. Comment expliquer que coopération et mutualité, sours de l'économie sociale, ne se soient pas alliées plus souvent? L'analyse des facteurs théoriques, historiques et juridiques qui caractérisent leurs points communs et leurs différences est nécessaire, pour mettre en perspective l'originalité de cette expérience. Les dirigeants socialistes de la coopérative d'alimentation La Fraternelle de Saint-Claude sont devenus acteurs de l'action sanitaire et sociale dans le Haut-Jura et ont fondé des structures mutualistes. Le facteur juridique a joué un rôle essentiel dans cette évolution structurelle.

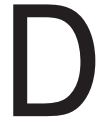

ans l'histoire de l'économie sociale française, l'alliance d'une coopérative et d'une mutuelle est un phénomène peu fréquent. Bien quappartenant à cette même mouvance idéologique qui souhaite apporter des solutions aux conséquences économiques et sociales de la révolution industrielle, coopération et mutualité évoluent séparément et ne se rapprochent qu'exceptionnellement. L'une se préoccupe d'alléger le poids économique dans la vie quotidienne de la population, l'autre, celui des aléas de la vie. Bien sûr, coopérateurs et mutualistes se côtoient, mais leurs organisations œuvrent indépendamment l'une de l'autre.

Le département du Jura nous offre l'exemple d'une expérience unique d'alliance très étroite entre une coopérative de consommation et une société de secours mutuels, La Fraternelle et la Mutuelle de la maison du peuple, à Saint-Claude. C'est une particularité à la fois locale et nationale et une exception dans l'histoire de la mutualité et de la coopération en France. Cette expérience de l'économie sociale, connue sous le nom d'école de Saint-Claude, n'a pas fait l'objet d'une étude approfondie. L'analyse de cette exception est l'occasion de s'interroger sur les éléments historiques, juridiques et idéologiques qui séparent ou rapprochent 
(1) Archives de la mutuelle Amellis (Saint-Claude); archives de la maison du peuple (Saint-Claude); archives municipales de SaintClaude; archives de la Mutualité française Jura (Lons-le-Saunier) archives départementales du Jura (Desgré, Mélo, 2010). coopération et mutualité. Comment expliquer, en effet, que ces deux sœurs de l'économie sociale ne se soient pas alliées plus souvent? Cette étude se fonde sur le dépouillement des différentes archives mutualistes jurassiennes et de celles de la maison du peuple de Saint-Claude ${ }^{(1)}$, mais aussi sur l'analyse des régimes juridiques des coopératives et des sociétés de secours mutuels. L'apport de l'histoire du droit est essentiel à la compréhension de l'articulation de leurs rôles.

Le Jura est un département montagneux, rural, aux hivers rigoureux, longtemps resté à l'écart des grands axes de communication. Dans ce paysage de monts, de vals, de crêtes et de plateaux, les rudes conditions d'existence sont adoucies par une forte tradition communautaire. Les années 1850-1870 marquent un tournant décisif: désenclavement par l'ouverture des voies ferrées et l'amélioration des routes vicinales, modernisation des pratiques agricoles, développement de l'élevage, spécialisation laitière et fromagère, essor de l'industrie métallurgique et spécialisation dans la fabrication de la pipe à Saint-Claude. Devenu terre d'élection de la gauche radicale, le Jura s'industrialise et s'urbanise. C'est dans la ville de Saint-Claude, à la fin du XIX siècle, qu'est mise en pratique cette voie nouvelle de l'économie sociale. L'école de SaintClaude se caractérise notamment par le rapprochement de la coopération et de la mutualité. L'analyse des problématiques d'une économie sociale naissante vient mettre en perspective cette expérience locale et son originalité.

\section{Les fondements d'une alliance : la coopération à vocation sociale}

(2) Cf. ministère du Commerce, Exposition d'économie sociale: enquête, Paris, Imprimerie nationale, 1887, p. 18.
Bien que les mouvements coopératifs et mutualistes soient issus du même creuset, ils se distinguent par des éléments théoriques, historiques et juridiques. A la fin du XIX ${ }^{\mathrm{e}}$ siècle, leur rapprochement est rendu possible par le politique. Cette vision globalisante, qui instrumentalise l'économie sociale, doit cependant tenir compte de certains obstacles juridiques.

\section{Des missions différentes, un objectif commun}

L'économie sociale, science née du foisonnement théorique et pratique d'un $\mathrm{XIX}^{\mathrm{e}}$ siècle marqué par les bouleversements politiques, économiques et sociaux, constitue un ensemble difficile à définir et à circonscrire. Les définitions sont nombreuses, qu'elles soient formelles ou matérielles. S'en tenir à l'énumération des composantes - comme c'est souvent le cas -, c'est-à-dire à la forme et aux expressions de l'économie sociale, ne renseigne pas sur les liens qui les unissent. Dans l'exposé des motifs de l'exposition sur l'économie sociale mise en place au sein de l'Exposition universelle de Paris de 1889, les pouvoirs publics optent pour une définition large: l'économie sociale représente "l'ensemble des sources $d u$ bien-être " (2). Coopératives et sociétés de secours mutuels y côtoient, en bonne place, les syndicats professionnels, les caisses 
de retraite, les caisses d'épargne, les habitations ouvrières, les cercles ouvriers, les sociétés de tempérance, les initiatives patronales, etc. Dans son ouvrage Economie sociale: les institutions du progrès au début du XXe siècle, Charles Gide (1847-1932), professeur de droit et d'économie politique et théoricien de cette toute jeune science sociale, présente une définition plus précise: l'économie sociale est l'étude « des rapports volontaires, contractuels, quasi contractuels ou légaux que les hommes forment entre eux en vue de s'assurer une vie plus facile " (Gide, 1905, p. 4). Ce qui rapproche mutualité et coopération, c'est donc la même volonté de lutter contre les effets de la révolution industrielle et du capitalisme libéral. Il s'agit d'apporter des solutions à la question sociale et à la misère. Le XIX ${ }^{e}$ siècle donne naissance à une profusion de théories et d'expérimentations nouvelles. De ce foisonnement émergent les idées coopératives et mutualistes sur fond de suspicion des autorités à l'égard de toute association.

Cet objectif et ce creuset expérimental communs se traduisent par une communauté de principes. Les deux mouvements sont fondés sur l'association, la sociabilité, la libre initiative collective, la démocratie, la solidarité et la promotion de l'individu. Assemblée délibérative, bureau exécutif, partage des dividendes sont au cour de ces organisations. A cet égard, la vie quotidienne des coopérateurs et celle des mutualistes présentent bien des similitudes. Ce mode d'organisation démocratique et libérale fonde, pendant longtemps, les inquiétudes du pouvoir, hostile à toute association qui pourrait s'ériger en mouvement de résistance. Rappelons que la liberté d'association reste fortement limitée par le Code pénal et qu'il faudra attendre la loi du $1^{\text {er }}$ juillet 1901 avant qu'elle ne devienne pleine et entière.

Cependant, chacun des deux mouvements assure une mission différente. La coopérative, en procurant des biens à moindre coût et en partageant des dividendes, améliore le quotidien des sociétaires, tandis que la mutualité prélève une épargne sur leur budget pour pouvoir faire face à d'éventuels aléas de la vie. Chacun se saisit d'un moment et d'un aspect spécifique de l'existence: l'ordinaire et l'extraordinaire, l'économique et le sanitaire, ce que Charles Gide résume ainsi, lorsqu' il aborde dans son Histoire des doctrines économiques celle, solidariste, de Léon Bourgeois: "Tandis que la mutualité et les assurances sociales ont pour but de réaliser la solidarité dans la manvaise fortune, en faisant participer au malheur des victimes tous les membres de la société mutuelle ou de la nation, les coopératives réalisent la solidarité en faisant participer tous les membres de la société à la bonne fortune créée par les plus dévoués d'entre eux" (Gide, Rist, 1922, p. 715). Il précise que les coopératives sont, des deux institutions, les plus éloignées de la notion de solidarité, ces liens d'interdépendance entre les hommes symbolisés par la formule "Chacun pour tous, tous pour chacun ", valeur fondamentale de l'économie sociale. Les sociétés de secours mutuels, en effet, sont quant à elles spécifiquement chargées de la lutte contre les risques sociaux. 
(3) Cf. Bulletin officiel du ministère de I'Intérieur, de l'Agriculture et du Commerce, Paris, Imprimerie administrative Paul-Dupont, 1852 p. 380 .

\section{Des histoires différentes, une alliance par le politique}

Issus du même creuset, les deux mouvements suivent, à compter du milieu du XIX ${ }^{\mathrm{e}}$ siècle, des chemins séparés par le droit et l'investissement de l'Etat. Il a pu exister auparavant des expériences de collaboration, telle cette société de secours mutuels lilloise, évoquée par le juriste Valleroux dans son ouvrage sur le mouvement coopératif, qui aux alentours de 1848 développe une activité coopérative par des achats groupés auprès des commerçants de la ville afin d'obtenir des prix pour ses adhérents (Valleroux, 1904). De même, les chambres syndicales ouvrières, sous le Second Empire, pouvaient mêler mutualité, coopération et résistance (Pottier, 1996; Soubiran-Paillet, 1999). Cependant, il faut attendre la fin du siècle pour que mutualité et coopération œuvrent main dans la main.

\section{La mutualité et l'Etat}

La mutualité plonge ses racines dans les corporations de métiers de l'Ancien Régime, mais elle prend sa forme aboutie dans le cadre administratif et juridique imposé par les pouvoirs publics à partir du Second Empire. A la crainte d'une mutualité de combat servant de refuge aux résistances ouvrières, l'Etat substitue une mutualité de notables fortement encadrée et contrôlée. Le rapport préalable au décret du 26 mars 1852, qui pose le statut et le cadre juridique de la mutualité, octroie aux sociétés de secours mutuels un rôle de garde-fou contre "l'esprit de désordre ", pour éviter de faire servir, "sous le masque de la bienfaisance ", "l'union des forces et des volontés à l'émeute et aux coalitions». Mais il s'agit également d'étendre les formes de prévoyance volontaire, protection contre les aléas de l'existence et les risques sociaux, car " il n'est pas pour le gouvernement de mission plus haute et plus importante que de travailler au bien-être des populations laborieuses, de diminuer leurs chances de malaise et de souffrance et de leur faciliter, après un long travail, le repos et une vieillesse honorée ${ }^{(3)}$ ". A partir de là, au gré des évolutions législatives, la mutualité se construit, se développe, se structure. Placée sous le contrôle de l'administration, elle se tient à distance du politique, du fait du principe de neutralité imposé par les pouvoirs publics. Elle assure auprès de l'Etat une mission d'intérêt général, celle de la prévoyance volontaire, tout en participant à l'encadrement et à la moralisation des masses. La Fédération nationale de la mutualité française (FNMF), fondée en 1902, assure le lien entre le monde mutualiste et l'Etat. La mutualité constitue un mouvement relativement homogène, un groupement de structures soumises à un régime juridique précis et assurant une même mission, tandis que la coopération est nettement plus hétérogène.

\section{La coopération divisée}

Al'inverse, les pouvoirs publics ne s'investissent pas autant dans l'encadrement et le développement de la coopération. Celle-ci est issue d'initiatives anglaises d'inspiration oweniste dont l'illustration la plus remarquable et la plus remarquée à l'époque est celle des ouvriers du textile de la petite 
ville de Rochdale rassemblés dans la Société des équitables pionniers, fondée en 1844 (Gueslin, 1998). Les initiatives sont diverses en fonction du mode de coopération: coopération de production, de consommation et de crédit. Avec le Second Empire, le mouvement bénéficie de la tolérance des pouvoirs publics, du soutien des notables, pour les coopératives de consommation plus particulièrement, et d'un support juridique fondé sur la loi du 24 juillet 1867 relative aux sociétés commerciales. Les liens juridiques et administratifs avec les pouvoirs publics restent faibles. Ce cadre léger explique en partie un développement paralysé par un manque d'organisation. Les coopératives vivent isolées les unes des autres. Les liens avec le politique s'affirment à la fin du siècle.

Aux origines de la structuration nationale du mouvement, à la Fédération des coopératives de consommation, fondée par Charles Gide en 1885 sur un principe de neutralité, s'oppose une Bourse des coopératives socialistes militante fondée en 1895 (Desroche, 1982; Pénin, 1997; Toucas-Truyen, 2005). Ce clivage exprime deux visions de la coopération: neutralité ou subordination vis-à-vis du politique.

Bien que partisan d'une "république coopérative ", Charles Gide se montre réticent envers certaines propositions de rapprochement. D'une part, il rejette toute utilisation pragmatique de la coopération par la mutualité, comme un rapprochement visant à permettre à une société de secours mutuels d'offrir des pensions de retraite à ses adhérents, ce qu’elle ne peut assumer seule en raison du coût trop élevé. Pour Gide et les partisans de l'école de Nîmes, il n'est pas question de transformer la coopération en «une simple annexe de la mutualité " par l'absorption des bonis de la coopérative. Les œuvres sociales ne doivent pas accaparer les bénéfices individuels, mais elles doivent être laissées à leur organe naturel que sont les sociétés de secours mutuels. D'autre part, le chef de file de la Fédération des coopératives de consommation se montre critique à l'égard d'une coopération militante subordonnée au socialisme, qu'il considère comme une sorte de patronage autoritaire pour le monde ouvrier. Il rejette une vision utilitariste de la coopération comme moyen d'atteindre le collectivisme.

De fait, apparaissent des velléités socialistes d'intégrer l'économie sociale et toutes ses composantes dans des projets de transformation sociale. Coopération, mutualité, syndicalisme, associationnisme doivent s’allier et collaborer à des fins politiques. Cette promotion de l'alliance des diverses composantes de l'économie sociale et du socialisme vient de Belgique. A partir de 1885, dans la région de Gand, sont créées des sociétés coopératives socialistes dont les bénéfices sont partiellement utilisés à la propagande politique et à la création de syndicats, de sociétés de secours mutuels et d'œuvres sociales et culturelles. La plus influente, le Vooruit, a été fondée grâce à un capital prêté par le syndicat des tisserands. A la boulangerie coopérative originelle viennent s’adjoindre boucherie, épicerie, magasins, thêâtre, café, pharmacie, caisse de secours pour soutenir les grèves, journal, bibliothèque, l'ensemble regroupé au sein d'une maison du peuple (Trémerel, 1894). 
Chaque coopérateur est affilié à une société de secours mutuels. Les bénéfices sont redistribués sous forme de jetons qui servent de monnaie d'échange contre les différents services offerts. Ce système oblige le sociétaire à dépenser au sein de la structure et à financer le projet politique. L'école belge (Ourman, 2001) influence une partie du mouvement coopératif, notamment la Bourse des coopératives socialistes fondée en 1900. Contrairement aux coopératives "bourgeoises" qui opèrent une simple répartition individuelle, les coopératives socialistes consacrent une partie des dividendes aux œuvres sociales.

\section{Des régimes juridiques différents, une alliance nécessaire}

C'est donc cette vision socialiste du mouvement coopératif qui incite au rapprochement avec le mouvement mutualiste. La coopération se donnant une mission sociale, même subordonnée à une mission politique, elle doit être à l'initiative d'œuvres sociales, telles que la fourniture de prestations en cas de maladie, d'accident ou de vieillesse à ses sociétaires. Cependant, concrètement, son régime juridique ne lui permet pas cette immixtion dans un champ réservé à la mutualité. Les questions juridiques peuvent être à l'origine de rapprochements pragmatiques, comme le montre l'évolution de la réglementation concernant l'autorisation de gérer une pharmacie, afin de pouvoir fournir à bas prix des médicaments aux sociétaires.

\section{Mutualistes et pharmaciens}

L'exercice de la profession de pharmacien est protégé et réglementé par la loi du 21 germinal an XI (11 avril 1803), dont l'article 25 interdit à toute personne n'étant pas munie d'un diplôme régulier de pharmacien le débit et la vente de médicaments (Coutant, 1902). A partir du Second Empire, par interprétation des textes, la jurisprudence étend cette possibilité aux hôpitaux, aux hospices, aux établissements de bienfaisance, aux sociétés de secours mutuels, mais interdit cependant son ouverture aux coopératives. A cette époque, la profession doit faire face à de nombreuses volontés d'immixtion dans son activité, déjà concurrencée par un " commerce inférieur » constitué d'épiciers, de droguistes, d'herboristes, etc. (Le Perdriel, 1862). Avant l'intervention du législateur, en 1898, c'est le juge qui, le premier, ouvre la voie à l'intervention mutualiste. Dans une décision du 17 juin 1880, la Cour de cassation étend l'exercice de la profession de pharmacien aux sociétés de secours mutuels, à condition que la gestion soit assurée par un pharmacien diplômé et que la vente soit limitée aux sociétaires (Barberet, 1904). La charte de la mutualité du $1^{\text {er }}$ avril 1898 ne fait que consacrer cette évolution et entériner l'élargissement de la mission mutualiste. L'article 8 de la loi permet le regroupement des sociétés de secours mutuels en unions, leur donnant la possibilité ainsi de réaliser en commun ce qu'elles ne peuvent réaliser seules, notamment la gestion d'œuvres sociales. La loi les autorise explicitement à créer des pharmacies. Dans sa décision du 22 décembre 1900, la Cour suprême confirme que rien dans la loi de 1898 n'interdit aux mutuelles de délivrer gratuitement 
des médicaments à leurs membres, dans une pharmacie installée par elles. En outre, rien n'exclut la possibilité de faire bénéficier les membres de la famille de l'adhérent de cette prestation.

\section{Coopérateurs ou pharmaciens}

Cette tolérance à l'égard de la mutualité s'explique par le caractère social de sa vocation. Il en va tout autrement pour les coopératives. Le caractère commercial de leur objet est incompatible avec la mission d'assistance des mutuelles. Les coopératives ne peuvent ouvrir une pharmacie à leur propre compte, mais elles ont, de plus, l'interdiction de s'associer dans ce but avec les mutuelles. Ainsi, en 1885, lorsque se forme à Tours un projet de création d'une coopérative de pharmacie afin de procurer aux adhérents des médicaments à bon prix, les autorités administratives saisies du dossier se prononcent pour l'interdiction, dénonçant une extension aux établissements de bienfaisance déjà trop flexible et des différences de statut juridique et de pratiques entre sociétés de secours mutuels et coopératives. Dans sa décision du 27 avril 1885, le comité consultatif d'hygiène justifie en effet la tolérance exceptionnelle accordée à la mutualité par le caractère gratuit des opérations et le régime juridique spécial qui place les sociétés de secours mutuels sous une étroite surveillance administrative. Une coopérative ne peut pas redistribuer à ses adhérents des médicaments qu'elle aurait achetés en gros. La Cour de cassation, dans une décision du 22 avril 1901, casse un arrêt rendu par la cour d'appel de Rennes le 13 décembre 1898 déboutant le syndicat des pharmaciens de la Loire-Inférieure de sa demande de condamnation de la coopérative de consommation de Trignac. En l'espèce, la coopérative achète des médicaments à un pharmacien et les redistribue à ses membres, qui payent avec des jetons. Selon les juges, "la société coopérative est, aux termes de l'article 53 de la loi du 24 juillet 1867, une personne civile distincte de ses membres, et qui acquiert et possède par ellemême; qu'en cédant au détail, moyennant un prix convenu, à ceux des sociétaires qui s'adresseraient à elle, des médicaments quielle a achetés avec ses ressources, elle leur en transmet la propriété, ce qui constitue à la fois une vente et un débit, en contravention avec la loi ".

Ces obstacles proprement juridiques rendent donc nécessaire un rapprochement entre coopérateurs et mutualistes, dès lors qu'une coopérative veut se donner une mission sociale. L'ensemble de ces éléments historiques, idéologiques et juridiques viennent expliquer l'expérience novatrice et unique menée par les Jurassiens.

\section{Les coopérateurs-mutualistes de l'école de Saint-Claude}

La caractéristique principale de la mutualité jurassienne réside dans le lien étroit entre coopération et mutualité. C'est au sein de la société d'alimentation La Fraternelle de la ville de Saint-Claude qu'émerge la volonté 
de donner une vocation sociale à la coopération. Les coopérateurs jurassiens, à la fois par conviction politique et sous la contrainte juridique, vont s'immiscer dans la mutualité en créant la Mutuelle de la maison du peuple, puis l'Union mutuelle du Haut-Jura.

\section{La Fraternelle: une coopérative à vocation sociale}

La société d'alimentation La Fraternelle naît en 1880, à l'initiative des membres du Cercle ouvrier de Saint-Claude, avec pour objectif l'amélioration des conditions d'existence des ouvriers de la ville. A l'origine, il s'agit d'une coopérative de consommation de type classique, ni socialiste ni sociale, même si les statuts préparatoires prévoient que les bénéfices doivent être versés au fonds de réserve de la société et non entièrement redistribués. La vocation sociale de La Fraternelle ne s'impose que plus tard. En 1896, des socialistes en prennent la direction. Au cours des années suivantes, ils semblent fortement influencés par l'expérience belge, qui devient un modèle d'organisation. Une alliance entre coopération et mutualité se profile. Le 8 novembre 1896, l'assemblée générale adopte les statuts d'une nouvelle société: l'association d'alimentation, de production, de prévoyance, de secours et de retraite La Fraternelle. Toute répartition individuelle des bénéfices est supprimée au profit d'une caisse sociale destinée à verser aux sociétaires des pensions de retraite et de secours en cas de maladie. La société devient à la fois coopérative d'alimentation et de production et organisme de prévoyance. A la mission coopérative classique, "l'approvisionnement, l'exploitation ou la vente de tous les objets de consommation, d'habillement, etc. " et "la production de tous articles", s'ajoute désormais "l'institution de tous services de solidarité quielle jugera bon d'entreprendre sur simple décision de l'assemblée générale " (art. 2). Les sociétaires ne perçoivent plus aucun bénéfice. De plus, ils ne peuvent bénéficier des nouvelles prestations sociales que s'ils dépensent une somme annuelle minimale au sein de la coopérative. Face à ce brusque virage idéologique, la crainte des oppositions internes entraîne l'adoption d'articles "spéciaux, irrévocables et irrévisables (sic) " qui déclarent "nulle et non avenue " toute proposition de modification ou de révision tendant à revenir sur la constitution et le partage de ce capital social (art. 4). Le lien étroit avec le socialisme municipal permet même d'envisager une protection radicale de celui-ci. Les statuts prévoient que la commune de Saint-Claude peut s'en emparer dans le cas où une telle modification serait adoptée à l'unanimité par l'assemblée générale. La municipalité pourrait dès lors constituer un service public ou une œuvre de bienfaisance (art. 5).

\section{La maison du peuple}

Cette expérience innovante donne naissance à "l'école de SaintClaude ", comme la nomme Charles Gide en 1902 lors d'une visite des organisations ouvrières de la région (Dreyfus, 2000). Il désigne ainsi une coopérative dont les fruits ne sont pas redistribués, mais alimentent des actions sociales au bénéfice des sociétaires. La coopération devient 
subordonnée à la prévoyance obligatoire et au politique. La Fraternelle rassemble bientôt, sous le toit d'une vaste maison du peuple, parti socialiste, syndicat et coopérative (Mélo, 1995). Cependant, cette coopérative originale doit rapidement muter et donner naissance à une société de secours mutuels, afin de se conformer aux exigences juridiques et de poursuivre le développement de sa vocation sociale. Si les sociétaires peuvent bénéficier de prestations, de pensions, sa marge de manœuvre reste délimitée par son statut juridique.

\section{Une coopérative et une société de secours mutuels: la Mutuelle de la maison du peuple}

Coopération et mutualité ne constituent nullement deux mondes étrangers l'un à l'autre. Coopérateurs et mutualistes, en tant qu'acteurs, se côtoient naturellement, comme ils côtoient les syndicalistes, les politiques et tous les animateurs de la vie locale, certains étant dotés de plusieurs casquettes. Ainsi, par leur participation aux instances locales et départementales, nombre de coopérateurs jurassiens sont également impliqués dans l'histoire mutualiste, motivés qu'ils sont par leur vision sociale. Des liens personnels existent et se tissent entre les deux mouvements.

\section{L'union mutualiste départementale}

Les coopérateurs engagés de Saint-Claude participent activement à la création de l'union mutualiste départementale, fondée le 6 mars 1904, et tentent d'y imposer leur projet social. Ainsi, ils obtiennent que soit inscrite dans les objectifs de la nouvelle instance la création de pharmacies comme l'un des buts d' "humanité" à atteindre par la mutualité. Mais des divergences partisanes se font jour entre ces militants et un monde mutualiste contraint à la neutralité, incitant à la création d'une union concurrente dans la circonscription de Saint-Claude, seule base juridique permettant la création et la gestion d'une pharmacie.

Lorsque les coopérateurs décident d'étendre leur action sociale à la gestion d'une pharmacie, sur le modèle belge, l'orientation mutualiste s'impose. Le lien structurel entre coopération et mutualité se resserre alors. En juillet 1907, les dirigeants de La Fraternelle incitent les administrateurs des sociétés de secours mutuels de la ville de Saint-Claude à s'unir pour l'ouverture et la gestion d'une pharmacie. Entre temps, la coopérative se prépare à la séparation des services " consommation" et "prévoyance». La Fraternelle ne peut plus mener seule de front ces deux missions. D'une part, pour répondre aux exigences juridiques, il est devenu nécessaire de créer une société de secours mutuels et de distinguer les deux gestions. D'autre part, cette création doit donner une base légale à une future union mutualiste, seule habilitée à gérer une pharmacie. Les coopérateurs militants doivent d'abord créer une société de secours, pour ensuite fonder une union de sociétés de secours mutuels et pouvoir ainsi atteindre leur objectif. 
Un hybride mutualiste et coopératif non reconnu par la préfecture Le 29 septembre 1907 se tient l'assemblée générale constitutive de la Mutuelle de la maison du peuple de Saint-Claude. La structure est une société de secours mutuels d'un nouveau type, une combinaison de règles mutualistes classiques - notamment en ce qui concerne les prestations, l'organisation et le fonctionnement - et de règles coopératives. Elle est alimentée non par le versement des cotisations, mais par celui d'une partie des bénéfices engendrés par La Fraternelle. Le lien entre les deux structures est étroit: seuls les adhérents de la coopérative peuvent être admis. Une fois encore, le bénéfice des prestations est subordonné à un certain volume de dépenses au sein de la coopérative et le seuil d'achat minimum est fixé en fonction du statut familial. Les prestations sont distribuées "en nature de marchandises prises dans les caves ou les magasins de La Fraternelle" ou en "objets d'alimentation", au moyen de jetons représentant la valeur des secours alloués (art. 14). La société se démarque également par son ouverture et son caractère familial: admission des femmes et des enfants, prise en charge du risque maternité, participation des mineurs aux assemblées générales et vote pour les jeunes de 16 ans révolus.

Cependant, certains de ces aspects novateurs dérogent à la règle et les statuts ne sont pas validés par l'autorité préfectorale. Des modifications doivent être apportées pour permettre l'inscription de la Mutuelle de la maison du peuple au registre des sociétés du département, notamment en matière de gestion et de financement. Le 26 avril 1908, les nouveaux statuts corrigés sont adoptés en assemblée générale.

\section{Une distinction obligée, une collaboration étroite}

Désormais, à l'instar de tout mutualiste, l'adhérent verse une cotisation et perçoit des secours en argent. L'apport de la coopérative devient une simple subvention. Le lien étroit subsiste toutefois, puisque les autorités acceptent que l'adhésion soit réservée exclusivement aux sociétaires de La Fraternelle (art. 5). De même, il est prévu que les éventuels bénéfices de la mutuelle soient annuellement laissés en dépôt à la coopérative sur un compte spécifique (art. 22).

A partir de cette création, il n'y a plus de confusion des genres entre activités coopératives et mutualistes, mais une collaboration étroite entre $\mathrm{La}$ Fraternelle et la Mutuelle de la maison du peuple, hébergées sous le même toit et animées par les mêmes personnalités.

\section{Une coopérative à la tête du mouvement mutualiste: I'Union mutuelle du Haut-Jura}

Une fois posée la base juridique et administrative nécessaire, reste à fonder la structure indispensable à la gestion d'une pharmacie. Le 13 octobre 1907 est créée l'Union mutuelle du Haut-Jura, qui regroupe des sociétés de secours mutuels de la région de Saint-Claude. Les statuts sont adoptés à l'unanimité et, aux termes de l'article premier, « il est fondé à Saint-Claude 
entre les sociétés qui adhéreront aux présents statuts une union mutuelle spéciale qui prend le nom de Pharmacie mutualiste de Saint-Claude ». Les fondateurs, représentants de mutuelles locales interprofessionnelles ou de coopératives, déclarent "vouloir avant tout accomplir une cuvre de solidarité sociale non limitée ». Pour eux, il ne s'agit que d'une première étape précédant l'établissement d'un dispensaire et la mise en place de consultations gratuites, de colonies d'enfants à la montagne et de toutes structures sanitaires "propres à lutter contre les maladies, les infirmités ou les fléaux qui déciment avec toujours plus d'intensité les familles ouvrières ». La pharmacie est officiellement ouverte le $1^{\text {er }}$ janvier 1908 dans les locaux de la maison du peuple. L'Union mutuelle du Haut-Jura en assure la direction et la gestion et compte alors vingt-deux sociétés de secours mutuels. La pharmacie doit permettre de procurer aux membres des sociétés adhérentes et à leurs familles des médicaments de première qualité à des conditions préférentielles. Les frais sont facturés directement aux sociétés adhérentes. L'offre s'étend également aux autres mutualistes de Saint-Claude et des cantons voisins, qui peuvent adhérer individuellement. Dans le respect de la législation, un pharmacien gérant est recruté sur diplôme et capacité. Il est responsable de la gestion, de la bonne tenue de l'officine et des préparations pharmaceutiques. Dès son ouverture, le nombre des bénéficiaires est estimé à $40 \%$ de la population géographiquement concernée.

Les dirigeants de La Fraternelle sont les initiateurs de ce projet et ils deviennent les fers de lance de la mutualité dans la région du Haut-Jura. Leur vision sociale influe sur le destin de la mutualité jurassienne, qui se caractérise notamment par un investissement dans le développement des œuvres sanitaires et sociales, dont le point de départ est marqué par la création de la pharmacie. Par le biais de la Mutuelle de la maison du peuple et de l'Union mutuelle du Haut-Jura, le conseil d'administration de La Fraternelle peut élargir l'offre de services en faveur de ses sociétaires: création d'une caisse chirurgicale (1913), création d'une pouponnière (1920), service de radiologie (1928), service de prothèse dentaire (1929), dispensaire médical (1934), laboratoire d'analyses (1939).

L'alliance entre coopération et mutualité se fonde donc essentiellement sur la volonté de développer la mission sociale de la première par son immixtion dans le champ de la prévoyance et par le développement d'œuvres sanitaires et sociales en direction de la classe ouvrière. Il s'agit d'une coopération à engagement socialiste qui intègre la mutualité dans une vision globale de l'action militante à des fins de transformation sociale. La maison du peuple de Saint-Claude rassemble le parti, l'organe de presse politique, le syndicat, la coopérative, la mutuelle et les œuvres sociales, aux côtés du théâtre, du café, de la salle de sport et du cinéma. 
Il serait cependant plus judicieux de parler de "l'expérience " de Saint-Claude plutôt que de "l'école » de Saint-Claude, dans la mesure où son influence reste limitée à la sphère locale et où elle n'essaime pas au-delà du département. Seules quelques coopératives de la région prennent modèle sur La Fraternelle. Selon l'expression même de celui qui qualifie cette expérience d'"école », il s'agit d'" une brillante exception dans le mouvement coopératif " (Gide, s. d., p. 114). Cependant, bien que leur expérimentation soit restée marginale, les coopérateurs-mutualistes de Saint-Claude marquent l'histoire de l'économie sociale et proposent un modèle d'alliance entre ces deux mouvements. 


\section{Bibliographie}

Barberet J., 1904, Les sociétés de secours mutuels: commentaire de la loi du $1^{\text {er }}$ avril 1898, Paris, Berger-Levrault éditeurs.

Coutant P., 1902, Précis de législation de la pharmacie, Lyon, Storck.

Desgré S., Mélo A., 2010, Amellis, histoire de la mutualité dans le Jura, Saint-Claude, Amellis mutuelles éditions.

Desroche H., 1982, Charles Gide (1847-1932): trois étapes d'une créativité, coopérative, sociale, universitaire, Paris, Ciem.

Dreyfus M., 2000, "Charles Gide, l'école de Saint-Claude et La Fraternelle ", Recma, n 275-276, avril, p. 53-59.

Gide C., 1905, Economie sociale: les institutions du progrès au début du XXe siècle, librairie de la société du Recueil Sirey.

Gide C., s. d., Cours sur la coopération au Collège de France (décembre 1925-avril 1926), Paris, Association pour l'enseignement de la coopération.

Gide C., Rist C., 1922, Histoire des doctrines économiques depuis les physiocrates jusqu'à nos jours, Paris, librairie de la société du Recueil Sirey, $4^{e}$ édition.

Gueslin A., 1998, L'invention de l'économie sociale: idées, pratiques et imaginaires coopératifs et mutualistes dans la France du $X I X^{e}$ siècle, Paris, Economica.

Le Perdriel C., 1862, Du passé, du présent et de l'avenir de la pharmacie, Paris, Masson éditions.

Mélo A., 1995, Une maison pour le peuple à Saint-Claude (1880-1940), Saint-Claude, Editions de La Fraternelle.

Ourman D., 2001, « Les influences du socialisme belge sur le socialisme français: la coopération (1885-1914) », Recma, n 280.

Penin M., 1997, Charles Gide (1847-1932): l'esprit critique, Paris, L'Harmattan.

Pottier M.-L., 1996, De l'usage professionnel à la loi: les chambres syndicales ouvrières parisiennes de 1867 à 1884, Paris, L'Harmattan.

Soubiran-Paillet F., 1999, L'invention dusyndicat: itinéraire d'une catégorie juridique, Paris, Librairie générale de droit et de jurisprudence. Toucas-Truyen P., 2005, Les coopérateurs: deux siècles de pratiques coopératives, Michel Dreyfus (dir.), Paris, L'Atelier.

Tremerel G., 1894, Des sociétés coopératives de consommation à l'étranger et en France, Paris, $\mathrm{V}$. Giard et E. Brière éditeurs.

Valleroux H., 1904, La coopération, Paris, Librairie Victor Lecoffre, 1904. 